VOL. 50 (1994) [59-72]

\title{
CONTINUITY OF THE SUPERPOSITION OPERATOR ON ORLICZ-SOBOLEV SPACES
}

\author{
G. HARDY AND H.B. THOMPSON
}

We give sufficient conditions for a homogeneous superposition operator to be a continuous mapping between Orlicz-Sobolev spaces. This extends a result of Marcus and Mizel concerning mappings between Sobolev spaces.

\section{INTRODUCTION}

Let $\Omega$ be a domain in $\mathbb{R}^{N}$, let $\mathcal{M}(\Omega)$ be the space of real measurable functions defined on $\Omega$, and, for a Borel measurable function $f: \mathbb{R} \rightarrow \mathbb{R}$, define the homogeneous superposition operator $T_{f}: \mathcal{M}(\Omega) \rightarrow \mathcal{M}(\Omega)$ by

$$
T_{f} u=f \circ u, \quad u \in \mathcal{M}(\Omega) .
$$

In [9], Marcus and Mizel show that (under certain conditions) $T_{f}$ is continuous as an operator from the Sobolev space $W^{1, p}(\Omega)$ to the Sobolev space $W^{1, r}(\Omega)$. Here we consider the continuity of $T_{f}$ on Orlicz-Sobolev spaces, and show that results analogous to those of Marcus and Mizel (for $1<\boldsymbol{p}<\infty$ ) hold for Orlicz-Sobolev spaces.

We remark that in both [5] and [8], results were obtained for the non-homogeneous superposition operator $T_{f}$ acting on vector-valued functions $u=\left(u_{1}, \cdots, u_{m}\right)$, where $T_{f}$ is defined by

$$
\left(T_{f} u\right)(x)=f(x, u(x))
$$

for $f: \Omega \times \mathbb{R}^{m} \rightarrow \mathbb{R}$. An example, given in Section 6, suggests that we cannot expect to obtain theorems on continuity of the type found here and in [9] in the general case.

\section{Preliminaries}

OrLicz Spaces. We shall use the properties of $N$-functions and Orlicz spaces as given in [7]. A brief summary of most of the definitions and theorems we need can also be found in [2]. To set the notation, and for later reference, we list a few properties below.

(i) An $N$-function $M$ satisfies the $\Delta_{2}$-condition (or $M \in \Delta_{2}$ ) if there exists a constant $u_{0} \geqslant 0$ and a real-valued function $k_{M}$ such that

$$
M(l u) \leqslant k_{M}(l) M(u) \text { for } u \geqslant u_{0}, \quad l>1 .
$$

Received 27th September, 1993.

Copyright Clearance Centre, Inc. Serial-fee code: 0004-9729/94 \$A2.00+0.00. 
(ii) If $M$ is an $N$-function, the Orlicz Space $L_{M}=L_{M}(\Omega)$, is the set of all functions $u$, measurable on $\Omega$, such that there exists a constant $\lambda>0$ such that $\int_{\Omega} M(\lambda u)<\infty$.

(iii) We shall use either the Luxemburg norm

$$
\|u\|_{M}=\|u\|_{M, \Omega}=\inf \left\{\lambda>0: \int_{\Omega} M(u / \lambda) \leqslant 1\right\}
$$

or the equivalent Orlicz norm

$$
\|u\|_{M}=\sup _{\int_{\Omega} \tilde{M}(v) \leqslant 1}\left\{\int_{\Omega}|u v|\right\}
$$

according to convenience. (Here $\widetilde{M}(v)=\sup _{u \geqslant 0}\{u|v|-M(u)\}$ denotes the $N$-function complementary to $M$.)

We have the following expressions for the norms of the characteristic function of a measurable set $A \subset \Omega$ :

$$
\begin{aligned}
\left\|\chi_{A}\right\|_{P, \Omega} & = \begin{cases}0, & \text { if } m_{N}(A)=0 \\
m_{N}(A) \widetilde{P}^{-1}\left[1 / m_{N}(A)\right], & m_{N}(A) \neq 0\end{cases} \\
\left\|\chi_{A}\right\|_{P, \Omega} & = \begin{cases}0, & \text { if } m_{N}(A)=0 \\
1 / P^{-1}\left[1 / m_{N}(A)\right], & m_{N}(A) \neq 0\end{cases}
\end{aligned}
$$

where $m_{N}(\cdot)$ denotes Lebesgue measure in $\mathbb{R}^{N}$.

(iv) Let $P$ and $M$ be $N$-functions, where $M \in \Delta_{2}$.

( $\alpha$ ) Suppose $P \circ M^{-1}$ is an $N$-function. If $u \in L_{P}(\Omega)$, then $M \circ u \in L_{P \circ M^{-1}}(\Omega)$, and

$$
\|M(u) \mid\|_{P \circ M^{-1}} \leqslant \text { const }\left(1+k_{M}\left(\|u\|_{P}\right)\right) .
$$

If further $P \circ\left(M^{\prime}\right)^{-1}$ is an $N$-function, and $M^{\prime}$ is strictly increasing, then

$$
\|\| M^{\prime}(u) \|_{P \circ\left(M^{\prime}\right)^{-1}} \leqslant \text { const }\left(1+k_{M^{\prime}}\left(\|u\|_{P}\right)\right) .
$$

(See [5].)

(v) If $R$ and $P$ are $N$-functions, we write $R \preccurlyeq P$ if there exist constants $u_{0}$ and $k$ such that $R(u) \leqslant P(k u)$ for all $u \geqslant u_{0}$; and $R \prec P$ if $\lim _{u \rightarrow \infty} R(\lambda u) / P(u)=0$ for all $\lambda>0$.

(vi) For $R \prec P$, the multiplicator space $L_{R}: L_{P}$ is defined as the set of all functions $v$ on $\Omega$ such that $u v \in L_{R}$ for all $u \in L_{P}$. We define the $N$-function $R: P$ by

$$
(R: P)(u)=\sup _{v \geqslant 0}\{R(u v)-\dot{P}(v)\}
$$


and then

$$
L_{R}: L_{P}=L_{R: P}
$$

( See [1].)

(vii) Let $P$ be a strictly convex $N$-function satisfying the $\Delta_{2}$-condition. If both $u_{n} \rightarrow u E_{\widetilde{P}}(\Omega)$-weakly and $\left\|u_{n}\right\|_{P, \Omega} \rightarrow\|u\|_{P, \Omega}$, then $\left\|u_{n}-u\right\|_{P, \Omega} \rightarrow 0$ as $n \rightarrow \infty$. (See [6].)

(viii) If $P \in \Delta_{2}$, then $u \in L_{P}(\Omega)$ has an absolutely continuous $L_{P}$ norm; that is, given, $\varepsilon>0$, there exists $\delta>0$ such that, for every measurable set $E \subset \Omega$ with $m_{N}(E)<\delta$, we have

$$
\left\|u \chi_{E}\right\|_{P, \Omega}<\varepsilon \text {. }
$$

(The same result holds with $\|\cdot\|$ instead of $\|\cdot\| \cdot$ )

Orlicz-Sobolev Spaces. We shall use the definitions and properties of OrliczSobolev spaces as given in [2].

(ix) The Sobolev conjugate $N$-function $P_{*}$ of an $N$-function $P$ is defined by

$$
P_{*}^{-1}(s)=\int_{0}^{|s|} P^{-1}(t) t^{-1-1 / N} d t
$$

where it is assumed that, if necessary, $P(t)$ is redefined for small values of $t$ (giving an equivalent $N$-function) so that

$$
\int_{0}^{1} P^{-1}(t) t^{-1-1 / N} d t<\infty
$$

(x) The Orlicz-Sobolev space $W^{1} L_{P}(\Omega)$ is defined as the set of all functions $u$ in $L_{P}(\Omega)$ whose distributional derivatives $\partial_{i} u$ also belong to $L_{P}(\Omega)$.

A norm $\|u\|_{1, P, \Omega}=\|u\|_{1, P}$ may be defined on $W^{1} L_{P}(\Omega)$ by

$$
\|u\|_{1, P}=\max \left\{\|u\|_{P},\left\|\partial_{1} u\right\|_{P}, \cdots,\left\|\partial_{N} u\right\|_{P}\right\}
$$

(xi) If $\Omega$ is a bounded domain in $\mathbb{R}^{N}$ satisfying the cone condition, we have the following continuous imbeddings:

(a) if $P_{*}^{-1}(\infty)=\infty, \quad W^{1} L_{P}(\Omega) \rightarrow L_{P_{*}}(\Omega)$;

(b) if $P_{*}^{-1}(\infty)<\infty, \quad W^{1} L_{P}(\Omega) \rightarrow L_{\infty}(\Omega) \cap C(\Omega)$.

(xii) Let $A(\Omega)$ denote the set of all functions $u$, measurable on $\Omega$, such that for almost all lines $\tau$ parallel to any coordinate axis $x_{i}, i=1, \cdots, N, u$ is locally absolutely continuous on $\tau \cap \Omega$. The Orlicz-Sobolev spaces $W^{1} L_{P}(\Omega)$ may be given an alternative characterisation in terms of the class $A(\Omega)$, as follows: 
Let $\Omega$ be a bounded domain in $\mathbb{R}^{N}$ with the cone property, and let $P$ be an $N$ function. A function $u$ defined on $\Omega$ is in $W^{1} L_{P}(\Omega)$ if and only if there exists $\widetilde{u} \in A(\Omega)$ such that

(a) $\tilde{u}=u$ almost everywhere in $\Omega$;

(b) $\partial \widetilde{u} / \partial x_{i} \in L_{P}(\Omega), \quad(i=1, \cdots, N)$.

Further $\partial \tilde{u} / \partial x_{i}=\partial_{i} u$ almost everywhere in $\Omega$. See [4] and [5].

\section{SUPERPosition Operators MapPing $W^{1} L_{P}(\Omega)$ INTO $W^{1} L_{P}(\Omega)$}

Let $\Omega$ be a bounded open subset of $\mathbb{R}^{N}$, let $f: \mathbb{R} \rightarrow \mathbb{R}$ be a uniformly Lipschitz function, and let $f^{*}: \mathbb{R} \rightarrow \mathbb{R}$ be any Borel function (which may be taken to be bounded) such that $f^{*}=f^{\prime}$ almost everywhere in $\mathbb{R}$. By using the arguments in Section 2 of [8] (replace their Lemma 1.5 by our 2(xii)) we can show that 2.1 in [9] holds for Orlicz spaces; that is,

$$
u \in W^{1} L_{P}(\Omega) \Rightarrow\left\{\begin{array}{c}
f \circ u \in W^{1} L_{P}(\Omega) \\
\partial_{i}(f \circ u)=\left(f^{*} \circ u\right) \partial_{i} u \quad \text { almost everywhere in } \Omega .
\end{array}\right\}
$$

We can now modify the proof of Theorem 1 in [9] to obtain the following:

Theorem 3.1. Let $\Omega$ and $f$ be as above, and let $P$ be a strictly convex $N$ function satisfying the $\Delta_{2}$-condition. Then the mapping $T_{f}: W^{1} L_{P}(\Omega) \rightarrow W^{1} L_{P}(\Omega)$ is continuous.

Proof: Let $u_{n} \rightarrow u$ in $W^{1} L_{P}(\Omega)$. By proceeding as in the proof of Theorem 1 $(p>1)$ in [9], we find that $f \circ u_{n} \rightarrow f \circ u$ in $L_{P}(\Omega)$ and that

$$
\partial_{i}\left(f \circ u_{n}\right) \rightarrow \partial_{i}(f \circ u) \quad E_{\widetilde{P}}(\Omega)-\text { weakly }
$$

If $f^{*}$ is the characteristic function of a Borel set, $g^{*}(t)=f^{*}(t)-1 / 2$, and $g(t)=$ 
$f(t)-t / 2$, then

$$
\begin{aligned}
\left\|\partial_{i}\left(g \circ u_{n}\right)\right\|_{P, \Omega} & =\sup _{\int \widetilde{P}(v) \leqslant 1} \int_{\Omega}\left|\left[g^{*}\left(u_{n}\right) \partial_{i} u_{n}\right] v\right| \\
& =\sup _{\int \tilde{P}(v) \leqslant 1} \int_{\Omega} \frac{1}{2}\left|\left(\partial_{i} u_{n}\right) v\right| \\
& =\frac{1}{2}\left\|\partial_{i} u_{n}\right\|_{P, \Omega} \rightarrow \frac{1}{2}\left\|\partial_{i} u\right\|_{P, \Omega} \\
& =\sup \int_{\Omega}\left|\left(\frac{1}{2} \partial_{i} u\right) v\right| \\
& =\sup _{n} \int_{\Omega}\left|\left[g^{*}(u) \partial_{i} u\right] v\right| \\
& =\left\|\partial_{i}(g \circ u)\right\|_{P, \Omega} .
\end{aligned}
$$

By 2.1 (vii), (3.1) and (3.2), $\partial_{i}\left(g \circ u_{n}\right) \rightarrow \partial_{i}(g \circ u)$ in $L_{P}(\Omega)$, and so $\partial_{i}\left(f \circ u_{n}\right) \rightarrow$ $\partial_{i}(f \circ u)$ in $L_{P}(\Omega)$, in this case.

The proof of Theorem 3.1 can now be completed as in the proof of Theorem 1 $(p>1)$ in $[9]$.

\section{Vitali's Theorem}

We shall need a version of Vitali's convergence theorem for Orlicz spaces. The following is adequate for our requirements.

Theorem 4.1. (Vitali). Let $\Omega \subset \mathbb{R}^{N}$ have finite measure, let $P$ be an $N$ function, let $\left\{f_{n}\right\}$ be a sequence in $L_{P}(\Omega)$, and let $f$ be a measurable function on $\Omega$. Then the following two conditions are sufficient for the convergence of $\left\{f_{n}\right\}$ to $f$ in $L_{P}(\Omega)$ :

(i) $\left\{f_{n}\right\}$ converges to $f$ in measure.

(ii) For each $\varepsilon>0$ there is a $\delta=\delta(\varepsilon)>0$ such that if $A \subset \mathbb{R}^{N}$ and $m_{N}(A)<\delta$, then

$$
\left\|f_{n} \chi_{A}\right\|_{P, \Omega}<\epsilon \quad \text { for all } n \in \mathbb{N}
$$

where $\mathbb{N}$ denotes the set of all positive integers.

If $P \in \Delta_{2}$, then (i) and (ii) are necessary for $\left\{f_{n}\right\}$ to converge to $f$ in $L_{P}(\Omega)$.

Proof: It is convenient to introduce the following notation: for $m \geqslant 1, n \geqslant 0$, and $\alpha>0$, let

$$
A_{m n}=A_{m n}(\alpha)=\left\{x \in \Omega:\left|f_{m}(x)-f_{n}(x)\right| \geqslant \alpha\right\}
$$


Observe that

$$
\left\|f_{m}-f_{n}\right\|_{P, \Omega} \geqslant \alpha\left\|\chi_{A_{m n}} \mid\right\|_{P, \Omega} .
$$

(a) Sufficiency. Suppose (i) and (ii) hold. Let $\varepsilon>0$, let $\delta$ be as in (ii), and let $\alpha=\varepsilon /\|\chi \Omega\|_{P, \Omega}$. From (i), there exists a positive integer $K=K(\varepsilon)$ such that if $m, n>K$

$$
m_{N}\left(A_{m n}\right)<\delta
$$

From (ii) and (4.3),

$$
\left\|f_{i} \chi_{A_{m n}} \mid\right\|_{P, \Omega}<\varepsilon
$$

for all $i \in \mathbb{N}$ and $m, n>K$.

Thus, using (4.2) and (4.4), we have

$$
\begin{aligned}
\left\|f_{m}-f_{n} \mid\right\|_{P, \Omega} & \leqslant\left\|\left(f_{m}-f_{n}\right) \chi_{\Omega \backslash A_{m n}} \mid\right\|_{P, \Omega}+\left\|f_{m} \chi_{A_{m n}}\right\|\left\|_{P, \Omega}+\right\| f_{n} \chi_{A_{m n}} \|_{P, \Omega} \\
& \leqslant \alpha\left\|\chi_{\Omega \backslash A_{m n}}\right\| \|_{P, \Omega}+\varepsilon+\varepsilon \leqslant 3 \varepsilon
\end{aligned}
$$

for $m, n>K$.

Therefore $\left\{f_{n}\right\}$ is Cauchy in $L_{P}(\Omega)$ and converges to a limit $\tilde{f}$ in $L_{P}(\Omega)$. From (4.2), with $f_{0}=\tilde{f}$, it follows that $\left\{f_{n}\right\}$ converges to $\tilde{f}$ in measure. Hence $\tilde{f}=f$ almost everywhere in $\Omega$, and so $\left\{f_{n}\right\}$ converges to $f$ in $L_{P}$, as required.

(b) Necessity. Now suppose $P \in \Delta_{2}$ and $\left\|f_{m}-f\right\|_{P, \Omega} \rightarrow 0$.

From (4.2), with $f_{0}=f$, it follows that $\left\{f_{m}\right\}$ converges to $f$ in measure. Thus (i) holds.

We now show that (ii) holds. Let $\varepsilon>0$, and choose $K$ so that

$$
\|\| f_{m}-f \|_{P, n}<\varepsilon / 2 \quad \text { for } m>K .
$$

From 2 (viii) we may choose $\delta>0$ so that

$$
\left\|f \chi_{A}\right\|_{P, \Omega}<\frac{\varepsilon}{2}
$$

and

$$
\|\| f_{m} \chi_{A} \|_{P, \Omega}<\varepsilon, \quad m=1,2, \cdots, K
$$

if $m_{N}(A)<\delta$. From (4.5) and (4.6),

$$
\begin{aligned}
\left\|f_{m} \chi_{A}\right\| \|_{P, \Omega} & \leqslant\left\|\left(f_{m}-f\right) \chi_{A}\right\|\left\|_{P, \Omega}+\right\| f \chi_{A} \|_{P, \Omega} \\
& <\varepsilon \quad \text { if } m>K .
\end{aligned}
$$

From (4.7) and (4.8), (ii) follows.

The following lemma is needed in our application of Vitali's theorem - Lemma 4.3. Lemma 4.3 is used in Theorem 5.4 . 
Lemma 4.2. If $\left\|u \chi_{A}\right\|_{Q, \Omega} \leqslant 1$ for measurable $A \subset \Omega$, and if $M$ and $Q$ are $N$-functions such that $Q \circ M^{-1}$ is an $N$-function, then

$$
\left\|M \circ \chi_{A}\right\|_{Q \circ M^{-1}, \Omega} \leqslant\left\|u \chi_{A}\right\|_{Q, \Omega}
$$

Proof: Since $\left\|u \chi_{A}\right\|_{Q, \Omega} \leqslant 1$,

$$
\left\|u \chi_{A}\right\|_{Q, \Omega}=\inf \left\{0<\lambda \leqslant 1: \int_{\Omega} Q\left(\frac{u \chi_{A}}{\lambda}\right) \leqslant 1\right\} .
$$

For $0<\lambda \leqslant 1, M(u / \lambda) \geqslant M(u) / \lambda$, so

$$
\begin{gathered}
Q \circ M^{-1} \circ M(u / \lambda) \geqslant Q \circ M^{-1}(M(u) / \lambda), \\
\int_{A} Q(u / \lambda) \geqslant \int_{A} Q \circ M^{-1}(M(u) / \lambda), \\
\inf \left\{0<\lambda \leqslant 1: \int_{A} Q(u / \lambda) \leqslant 1\right\} \geqslant \inf \left\{0<\lambda \leqslant 1: \int_{A} Q \circ M^{-1}(M(u) / \lambda) \leqslant 1\right\} .
\end{gathered}
$$

(4.9) follows readily from the last inequality.

LEMMA 4.3. Let $Q, M$, and $R$ be $N$-functions such that

(i) $Q \in \Delta_{2}$;

(ii) $Q \circ M^{-1}$ is an $N$-function;

(iii) $R \preccurlyeq Q \circ M^{-1}$.

Let $\Omega \subset \mathbb{R}^{N}$ have finite measure, and let $f: \mathbb{R} \rightarrow \mathbb{R}$ be a continuous function such that there exist constants $a, b$ and $c$ such that

(iv) $|f(t)| \leqslant c+a|t|+b M(t)$ for all $t \in \mathbb{R}$.

If $u_{n} \rightarrow u$ in $L_{Q}(\Omega)$, then $f\left(u_{n}\right) \rightarrow f(u)$ in $L_{R}(\Omega)$.

Proof: Since $u_{n} \rightarrow u$ in $L_{Q}(\Omega)$, by Theorem 4.1 there exists $\eta>0$ such that if $m_{N}(A)<\eta$ then

$$
\left\|u_{n} \chi_{A}\right\|_{A, \Omega} \leqslant 1 \quad \text { for all } n \in \mathbb{N} \text {. }
$$

From (iv),

$$
\begin{gathered}
\left\|f\left(u_{n}\right) \chi_{A}\right\|_{R, \Omega} \leqslant c\left\|\chi_{A}\right\|_{R, \Omega}+a\left\|u_{n} \chi_{A}\right\|_{R, \Omega} \\
+b\left\|M\left(u_{n}\right) \chi_{A}\right\|_{R, \Omega} .
\end{gathered}
$$

From Lemma 4.2, (4.11), and (iii), if $m_{N}(A)<\eta$

$$
\left\|M\left(u_{n}\right) \chi_{A}\right\|_{R, \Omega} \leqslant \text { const }\left\|u_{n} \chi_{A}\right\|_{Q, n} .
$$


Since (iii) implies that $R \preccurlyeq Q$, for $m_{N}(A)<\eta$, we have

$$
\left\|f\left(u_{n}\right) \chi_{A} \mid\right\|_{R, \Omega} \leqslant D\left[\left\|\chi_{A}\right\|_{Q, \Omega}+\left\|u_{n} \chi_{A}\right\|_{Q, \Omega}\right]
$$

for all $n \in \mathbb{N}$ and some constant $D$.

We may partition $\Omega$ into a finite number of subsets $\Omega_{i}$ such that $m_{N}\left(\Omega_{i}\right)<\eta$. From (4.12), $f\left(u_{n}\right) \in L_{Q}\left(\Omega_{i}\right)$, and hence $f\left(u_{n}\right) \in L_{Q}(\Omega)$.

Since $u_{n} \rightarrow u$ in measure and $f$ is continuous, $f\left(u_{n}\right) \rightarrow f(u)$ in measure.

We choose $\delta_{1}$ so that

$$
\left\|\chi_{A}\right\|_{A, \Omega}<\frac{\varepsilon}{2 D} \quad \text { for } m_{N}(A)<\delta_{1} .
$$

Using Vitali's theorem, we choose $\delta_{2}$ so that

$$
\left\|u_{n} \chi_{A} \mid\right\|_{P, \Omega}<\frac{\varepsilon}{2 D} \quad \text { for all } n \in \mathbb{N} \text { and } m_{N}(A)<\delta_{2} .
$$

Thus, by (4.12),

$$
\left\|f\left(u_{n}\right) \chi_{A}\right\|_{R, \Omega}<\varepsilon \quad \text { for all } n \in \mathbb{N},
$$

if $m_{N}(A)<\delta=\min \left(\eta, \delta_{1}, \delta_{2}\right)$.

By Vitali's theorem, $f\left(u_{n}\right) \rightarrow f(u)$ in $L_{R}(\Omega)$, as required.

\section{Superposition Operators Defined by Functions BOUNDED BY $N$-FunCTIONS}

We first give a lemma, corresponding to Lemma 3 in [9].

Lemma 5.1. Let $\Omega$ be a bounded domain in $\mathbb{R}^{N}$ satisfying the cone condition, and let $f: \mathbb{R} \rightarrow \mathbb{R}$ be a locally Lipschitz function. Let $P$ and $R$ be $N$-functions. Suppose that one of the following sets of conditions holds:

(a) $P_{*}^{-1}(\infty)<\infty$ and $R=P$;

or

(b) $P_{*}^{-1}(\infty)=\infty$ and there exists an $N$-function $M \in \Delta_{2}$ such that $M^{\prime}$ is strictly increasing and

$$
\left|f^{\prime}(t)\right| \leqslant a+b M^{\prime}(t)
$$

where $a$ and $b$ are constants. Suppose further that $P_{*} \circ\left(M^{\prime}\right)^{-1}$ and $P_{*} \circ M^{-1}$ are the principal parts of $N$-functions (completed, if necessary, by the procedure described in [5], page 16) and that

$$
\begin{gathered}
R \prec P, \\
R: P \preccurlyeq P_{*} \circ\left(M^{\prime}\right)^{-1}, \\
R \preccurlyeq P_{*} \circ M^{-1} \cdot \\
\text { If } u \in W^{1} L_{P}(\Omega), \text { then } f \circ u \in W^{1} L_{R}(\Omega)
\end{gathered}
$$


and

$$
\partial_{i}(f \circ u)=\left(f^{*} \circ u\right) \partial_{i} u \quad \text { almost everywhere in } \Omega
$$

Proof: Case (a) of the theorem follows from the Orlicz-Sobolev imbedding theorem $2(x i)(b)$.

Now suppose the hypotheses (b) hold. Let $v=f \circ u$. By 2(xii), there exists $\tilde{u} \in A(\Omega)$ such that $\tilde{u}=u$ almost everywhere in $\Omega$, and then $\tilde{v}=f \circ \tilde{u}=$ $v$ almost everywhere in $\Omega$. From (5.1), (5.2) and (5.3),

$$
\begin{aligned}
\left\|\frac{\partial \widetilde{v}}{\partial x_{i}}\right\| \|_{R, \Omega} & \leqslant\left\|a+b M^{\prime}(|\tilde{u}|) \frac{\partial \tilde{u}}{\partial x_{i}} \mid\right\|_{R, \Omega} \\
& \leqslant \text { const }\left[\left\|\frac{\partial \tilde{u}}{\partial x_{i}}\left|\left\|_{P, \Omega}+\right\| M^{\prime}(|\tilde{u}|)\right|\right\|_{R: P, \Omega}\left\|\frac{\partial \widetilde{u}}{\partial x_{i}}\right\| \|_{P, \Omega}\right] \\
& \leqslant \text { const }\left[\|\tilde{u}\|_{1, P, \Omega}+\left\|M^{\prime}(|\tilde{u}|)\right\|\left\|_{P_{*} \circ\left(M^{\prime}\right)^{-1}, \Omega}\right\| \tilde{u} \|_{1, P, \Omega}\right] .
\end{aligned}
$$

From the Orlicz-Sobolev imbedding theorem 2(xi)(a), $\|u\|_{P_{*}, \Omega}<\infty$, so by 2(iv), $\left\|M^{\prime}(|\tilde{u}|) \mid\right\|_{P_{*} \circ\left(M^{\prime}\right)^{-1, \Omega}}<\infty$. Thus we have

$$
\left\|\frac{\partial \bar{v}}{\partial x_{i}}\right\|\left\|_{R, \Omega}=\right\| \partial_{i} f(u) \|_{R, \Omega}<\infty .
$$

Integrating the inequality (5.1), we obtain

$$
|f(t)-f(0)| \leqslant a|t|+b M(t) .
$$

Therefore, using (5.2) and (5.4),

$$
\begin{aligned}
\|f(u)-f(0)\|_{R, \Omega} & \leqslant a\|u\|\left\|_{R, \Omega}+b\right\| M(u) \|_{R, \Omega} \\
& \leqslant \text { const }\left[\|u\|_{P, \Omega}+\|M(u)\|_{P_{*} \circ M^{-1}, \Omega}\right] \\
& <\infty
\end{aligned}
$$

from 2 (iv), because $\|u\|_{P_{*}, \Omega<\infty} \cdot(5.7)$ and (5.8) establish (5.5), and then (5.6) follows from $2(\mathrm{xii})$.

EXAMPLES OF $N$-FUNCTIONS SATISFYing THE CONDITIONS OF LEMMA 5.1 .

EXAMPLE 5.2. Suppose $q>0$, and for $1<r<p<N$, define $r: p$ by

$$
\frac{1}{r: p}=\frac{1}{r}-\frac{1}{p}
$$


and $p_{*}$ by

$$
\frac{1}{p_{*}}=\frac{1}{p}-\frac{1}{N}
$$

Now suppose that

$$
\frac{q}{p_{*}} \leqslant \frac{1}{r: p}
$$

Let $R(t)=1 / r|t|^{r}, P(t)=1 / p|t|^{p}$, and $M(t)=1 /(q+1)|t|^{q+1}$. Then $P_{*}^{-1}(\infty)=$ $\infty, R \prec P, M \in \Delta_{2}$ and $M^{\prime}(t)$ is strictly increasing for $t>0$. An elementary calculation (see 2(ix)) shows that

$$
(R: P)(t)=1 /(r: p)|t|^{r: p}
$$

From (5.9), we have

$$
R: P \preccurlyeq P_{*} \circ\left(M^{\prime}\right)^{-1}
$$

It follows from (5.9) that

$$
\frac{1}{r} \leqslant \frac{q+1}{p_{*}}
$$

from which we obtain

$$
R \preccurlyeq P_{*} \circ M^{-1}
$$

This example shows that our Lemma 5.1 contains Lemma 3.1 (for $r<p<N$ ) in [8] as a particular case. For $p>N$, and $r=p$, we obtain Marcus and Mizel's result by defining $P$ as before.

NoTE 1. There appears to be a typographical error in [9]. In the statement of Lemma 3 , part (b), the case $p<N$ " $1 / r \leqslant(q+1) / p$ " should read " $1 / r \geqslant(q+1) / p_{*}$ ".

Note 2. In the case of power functions (5.4) follows from (5.3). It would be interesting to know if the same holds in the general case.

Example 5.3. Suppose $M, P$, and $R$ are $N$-functions such that

(i) $M^{\prime}(u)$ is continuous and strictly increasing for $u>0$,

(ii) $M \in \Delta_{2}$,

(iii) $P_{*}^{-1}(\infty)=\infty$,

(iv) $R^{-1}(t)=M^{\prime} \circ P_{*}^{-1}(t) \cdot P^{-1}(t)$ for large $t$,

(v) $P_{*} \circ\left(M^{\prime}\right)^{-1}$ is an $N$-function.

Since $R \prec P$ is equivalent to $\lim _{t \rightarrow \infty}\left(P^{-1}(t)\right) /\left(R^{-1}(t)\right)=0,(5.2)$ follows from (iv). It was shown in [5] (Lemma 3.3) that (i), (iii), (iv) and (v) imply that

$$
\|u v\|_{R} \leqslant \text { const }\|v\|_{P_{*} \circ\left(M^{\prime}\right)^{-1}, \Omega}\|u\|_{P, \Omega}
$$


whence (see 2(vi))

$$
L_{P_{*} \circ\left(M^{\prime}\right)^{-1}}(\Omega) \subseteq L_{R: P}(\Omega)
$$

and so (see [7], Theorem 13.1)

$$
R: P \preccurlyeq P_{*} \circ\left(M^{\prime}\right)^{-1} .
$$

Thus (5.3) holds.

It was also shown in [5] that (i), (iii), (iv) and (v) imply that $P_{*} \circ M^{-1}$ is the principal part of an $N$-function and that $R_{*} \sim P_{*} \circ M^{-1}$. (5.4) then follows from the fact that $R \prec R_{*}$. (See [3], Lemma 4.14.)

We can construct particular cases of $N$-functions satisfying (i) - (v) of Example 5.3 by taking $M$ and $P$ to be power functions, as in Example 5.2. (iv) then defines $R$ as a power function.

It was shown in [5] that there exist $N$-functions satisfying (i) - (v), for which only $P$ was a power function: for $1<p<N$ define $P(t)$ by $P(t)=c|t|^{p}$ ( $c$ a constant), for $q>0$ define $M$ by $M(t)=|t|^{q+1}(\ln |t|+1)$ for large $t$ and define $R$ by (iv) for large $t$. For the details, see [5].

Theorem 5.4 below corresponds to Theorem 2 in [9].

THEOREM 5.4. Let $f, M, P, R$ and $\Omega$ be as in Lemma 5.1, and suppose further that $P \in \Delta_{2}$ and is strictly convex. Then the mapping $T_{f}: W^{1} L_{P}(\Omega) \rightarrow W^{1} L_{R}(\Omega)$ is continuous.

Proof: Let $f: \mathbb{R} \rightarrow \mathbb{R}$ be locally Lipschitz and $u_{n}$ converge to $u$ in $W^{1} L_{P}(\Omega)$. We need to show that

$$
T_{f} u_{n} \rightarrow T_{f} u \text { in } W^{1} L_{R}(\Omega)
$$

We consider the case $\left\|u_{n}\right\|_{L_{\infty}(\Omega)}$ is unbounded; the case $\left\|u_{n}\right\|_{L_{\infty}(\Omega)}$ is bounded follows similarly to that in Theorem 2 of [9].

Suppose that $f, M, P$ and $R$ are as in Lemma $5.1(\mathrm{~b})$, and that $P \in \Delta_{2}$ and is strictly convex.

Let $w \in W^{1} L_{P}(\Omega)$ and $c>0$. Following [9], we use the notation

$$
\begin{aligned}
S_{i} w & =\left(f^{*} \circ w\right) \partial_{i} w, \quad i=1, \cdots, N \\
A_{c}(w) & =\{x \in \Omega:|w(x)|>c\} \\
w_{c} & =h_{c} \circ w
\end{aligned}
$$

where $h_{c}(t)=t$ for $|t| \leqslant c, h_{c}(t)=+c(-c)$ for $t>c(<-c)$. Then

$$
\begin{aligned}
S_{i} w_{c} & =0 \quad \text { almost everywhere in } A_{c}(w) \\
S_{i} w_{c} & =S_{i} w \quad \text { almost everywhere in } \Omega \backslash A_{c}(w) .
\end{aligned}
$$


For the details, see the proof of Theorem 2 in [9].

Since $u_{n} \rightarrow u$ in $L_{P_{*}}(\Omega)$, Lemma 4.3 shows that $T_{f} u_{n} \rightarrow T_{f} u$ in $L_{R}(\Omega)$. By Theorem 3.1, $u_{n, c} \rightarrow u_{c}$ in $W^{1} L_{P}(\Omega)$, and so $T_{f} u_{n, c} \rightarrow T_{f} u_{c}$ in $W^{1} L_{P}(\Omega)$. This implies that

$$
S_{i} u_{n, c} \rightarrow S_{i} u_{c} \text { in } L_{R}(\Omega)
$$

because $R \prec P$.

Because $u_{n}$ converges in $W^{1} L_{P}(\Omega)$, the sequence $\left\|u_{n}\right\|_{P_{*}, \Omega}$ is bounded, from the Orlicz-Sobolev inequality, and therefore so is the sequence $\left\|M^{\prime}\left(u_{n}\right) \mid\right\|_{P_{*} \circ\left(M^{\prime}\right)^{-1}, \Omega}$, from 2(iv). Then

$$
\begin{aligned}
\left\|S_{i} u_{n, c}-S_{i} u_{n} \mid\right\|_{R, \Omega} & \leqslant\left\|S_{i} u_{n} \chi_{A_{c}\left(u_{n}\right)} \mid\right\|_{R, \Omega} \\
& \leqslant C_{1}\left\|a+b M^{\prime}\left(\left|u_{n}\right|\right)\right\|_{R: P, \Omega}\left\|\partial_{i} u_{n} \chi_{A_{c}\left(u_{n}\right)}\right\| \|_{P, \Omega} \\
& \leqslant C_{2}\left\|a+b M^{\prime}\left(\left|u_{n}\right|\right)\left|\left\|_{P_{*} \circ\left(M^{\prime}\right)^{-1}, \Omega}\right\|\right| \partial_{i} u_{n} \chi_{A_{c}\left(u_{n}\right)}\right\| \|_{P, \Omega} \\
& \leqslant C_{3}\left\|\partial_{i} u_{n} \chi_{A_{c}\left(u_{n}\right)}\right\|_{P, \Omega},
\end{aligned}
$$

where $C_{1}, C_{2}$ and $C_{3}$ are constants. Similar arguments show that

$$
\left\|S_{i} u_{c}-S_{i} u\right\|_{R, \Omega} \leqslant C_{4} \mid\left\|\partial_{i} u \chi_{A_{c}(u)}\right\|_{P, \Omega}
$$

where $C_{4}$ is a constant.

Let $\varepsilon>0$. By Vitali's Theorem and 2(viii) we may choose $\delta>0$ so that both

$$
C_{3}\left\|\partial_{i} u_{n} \chi_{A}\right\|_{P, \Omega}<\varepsilon
$$

and

$$
C_{4}\left|\left\|\partial_{i} u \chi_{A} \mid\right\|_{P, \Omega}<\varepsilon\right.
$$

if $m_{N}(A)<\delta$. As shown in [9], we may choose $n(\delta)$ and $c(\delta)$ so that $m_{N}\left(A_{c}(u)\right)<\delta$ and $m_{N}\left(A_{c}\left(u_{n}\right)\right)<\delta$ for $n \geqslant n(\delta), c \geqslant c(\delta)$. We thus have

$$
\left\|S_{i} u_{n, c}-S_{i} u_{n}\right\|_{R, \Omega}<\varepsilon
$$

and

$$
\left\|S u_{c}-S u\right\|_{R, n}<\varepsilon
$$

for $n \geqslant n(\delta)$ and $c \geqslant c(\delta)$.

(5.12), (5.15) and (5.16) show that

$$
S_{i} u_{n} \rightarrow S_{i} u \text { in } L_{R}(\Omega)
$$

The theorem follows from (5.10) and (5.17). 


\section{NONHOMOGENEOUS SUPERPOSITION OPERATORS}

It is natural to ask if any of the continuity results for homogeneous superposition operators extend to the nonhomogeneous case. We construct $f: \mathbb{R}^{2} \rightarrow \mathbb{R}$ which is uniformly Lipschitz continuous and a sequence $u_{i}$ converging to 0 in $W^{1, \infty}[-\pi, \pi]$ such that $T_{f}\left(u_{i}\right)$ does not converge in $W^{1,2}[-\pi, \pi]$ to $T_{f}(0)=0$, where

$$
T_{f}(u)(x)=f(x, u(x))
$$

Then $T_{f}: W^{1, \infty}[-\pi, \pi] \rightarrow W^{1,2}[-\pi, \pi]$. If $f$ is independent of $x$, then $T_{f}$ is continuous in $W^{1,2}[-\pi, \pi]$. In view of these observations it is difficult to see what necessary and sufficient conditions can be given for nonhomogeneous operators.

EXAMPLE 6.1. For each natural number $n$ set $f\left(x, 2^{-n}\right)=2^{-n} \sin 2^{n} x$ and $f(x, 0)=0$. Thus $\left|f_{x}\left(x, 2^{-n}\right)\right|=\left|\cos 2^{n} x\right| \leqslant 1$ for all $n$. Assuming $n<m$

$$
\begin{aligned}
\left|f\left(x, 2^{-n}\right)-f\left(x, 2^{-m}\right)\right| & \leqslant\left|2^{-n} \sin 2^{n} x\right|+\left|2^{-m} \sin 2^{m} x\right| \\
& \leqslant 2^{-n+1} \\
& \leqslant 4\left|2^{-n}-2^{-m}\right| \\
\text { and }\left|f\left(x, 2^{-n}\right)-f(x, 0)\right| & =\left|2^{-n} \sin 2^{n} x\right|=\left|2^{-n}-0\right| .
\end{aligned}
$$

Thus $f$ is uniformly Lipschitz on the closed set

$$
G=\left\{(x, y) \in[-\pi, \pi] \times \mathbb{R}: y=2^{-\pi}, \text { some } n \text {, or } y=0\right\}
$$

Extend $f$ to $[-\pi, \pi] \times \mathbb{R}$ with the same Lipschitz constant. Let $u_{n}=2^{-n}$ so $u_{n}$ converges to 0 in $W^{1, \infty}[-\pi, \pi]$. We show $T_{f}\left(u_{n}\right)$ does not converge in $W^{1,2}[-\pi, \pi]$ to $T_{f}(0)=0$. Now $\left|f\left(x, u_{n}\right)\right| \leqslant 2^{-n}$ and $\frac{d}{d x} f\left(x, u_{n}\right)=\sin 2^{n} x$ so $\frac{d}{d x} f\left(x, u_{n}\right)$ converges weakly to 0 in $L_{2}[-\pi, \pi]$ and $\left\|\frac{d}{d x} f\left(x, u_{n}\right)\right\|_{L_{2}}=\pi$. Thus $\frac{d}{d x} f\left(x, u_{n}\right)$ does not converge in $L_{2}[-\pi, \pi]$ and thus $T_{f}\left(u_{n}\right)$ does not converge in $W^{1,2}[-\pi, \pi]$.

REMARK. This counterexample also shows that necessary and sufficient conditions for the continuity of homogeneous superposition operators on vector valued Sobolev spaces are likely to be complicated. Just set $T_{f}\left(u_{1}, u_{2}\right)(x)=f\left(u_{1}(x), u_{2}(x)\right)$. Thus $T_{f}$ : $W^{1, \infty}[-\pi, \pi] \times W^{1, \infty}[-\pi, \pi] \rightarrow W^{1,2}[-\pi, \pi]$. Then $f$ is uniformly Lipschitz continuous and $\left(u_{1 n}, u_{2 n}\right)=\left(x, 2^{-n}\right)$ converges in $W^{1, \infty}[-\pi, \pi] \times W^{1, \infty}[-\pi, \pi]$ to $(x, 0)$ but $T_{f}\left(u_{1 n}, u_{2 n}\right)$ does not converge to $T_{f}(x, 0)$.

\section{References}

[1] J. Appell and G. Hardy, 'On products of Sobolev-Orlicz spaces', Bull. Austral. Math. Soc. 42 (1990), 427-437. 
[2] T.K. Donaldson and N.S. Trudinger, 'Orlicz-Sobolev spaces and imbedding theorems', $J$. Funct. Anal. 8 (1971), 52-75.

[3] J-P. Gossez, 'Non-linear elliptic boundary problems for equations with rapidly (or slowly) increasing coefficients', Trans. Amer. Math. Soc. 190 (1974), 163-205.

[4] G. Hardy, 'Extensions of theorems of Gagliardo and Marcus and Mizel to Orlicz spaces', Bull. Austral. Math. Soc. 23 (1981), 121-138.

[5] G. Hardy, 'Nemitsky operators between Orlicz-Sobolev spaces', Bull. Austral. Math. Soc. 30 (1984), 251-269.

[6] J. Hudzik and M. Mastylo, 'Strongly extreme points in Köthe-Bochner Spaces', Rocky Mountain J. Math. 23 (1993), 899-909.

[7] M.A. Krasnosel'skiǐ and Ya.B. Rutickiǐ, Convex functions and Orlicz spaces, (translated from the first Russian edition by Leo F. Boron) (Noordhoff, Groningen, 1961).

[8] M. Marcus and V.J. Mizel, 'Absolute continuity on tracks and mappings of Sobolev spaces', Arch. Rational Mech. Anal. 45 (1972), 294-320.

[9] M. Marcus and V.J. Mizel, 'Every superposition operator mapping one Sobolev space into another is continuous', J. Funct. Anal. 33 (1979), 217-229.

Department of Mathematics

The University of Queensland

Queensland 4072

Australia 